\title{
Evidence on Time-Varying Factor Models for Equity Portfolio Construction
}

\author{
Markus Ebner ${ }^{1}$ and Thorsten Neumann ${ }^{2}$ \\ 1 Union PanAgora Asset Management, Frankfurt, Germany \\ 2 Union Investment Institutional, Frankfurt, Germany, \\ Thorsten. Neumann@union-investment. $\mathrm{de}^{\dagger}$
}

\section{Introduction}

Many applicationers derive the variance-covariance matrix (VCM) for meanvariance optimization from some risk model or apply a simple historical estimate. A common problem to these approaches is the stability of the variancecovariance matrix. In turbulent market phases risk estimates from various risk models are well known to be unreliable. One reason for their poor risk forecasting ability is the fact that financial markets are subject to substantial structural change, applied risk models do not account for. In our paper we account for structural changes by deriving VCMs from time-varying estimates of the single factor model, i.e., the market model. We demonstrate the advantages of this approach with respect to risk estimation, portfolio selection and investment performance by means of simulated trading strategies.

The problem of choosing the adequate risk model has come in mind of scientific researchers and practioners only recently. While research has focused on forecasting returns for a long time there is a lack of evidence in evaluating the performance of different risk models and the consequences for portfolio optimization. Next to the well known sensitivity of the mean-variance optimization with respect to assumed expected returns the benefits promised by this approach also heavily depend on the accuracy in estimating the VCM (see, for example, [1] and [4]). Given the well known difficulty of estimating expected returns the most important improvement on MV optimization can be made in the VCM estimation which is mainly based on financial econometrics. However, on the performance of alternative risk models and optimization procedures there is only limited scientific evidence, such as $[3,9,10,13,18]$ among others.

The capital asset pricing model (CAPM) due to [17] and [14] assumes stock returns to be a linear function of a single factor, namely the market return. Stock betas, i.e., stock return elasticities with respect to the market

\footnotetext{
${ }^{\dagger}$ Corresponding author: Thorsten Neumann, Union Investment Institutional
} 
return, have been widely used to evaluate systematic risk, i.e., the return risk associated with market movements.

When estimating the CAPM it is common practice to assume stock betas to be invariant over time. However, this stability assumption has been questioned and a considerable amount of empirical evidence reports important beta variation over time (see among others, [2, 5, 8, 12, 16, 19], as well as [7]). Beta variation over time goes hand in hand with unstable correlations among stock returns and time-varying VCMs. This might have serious consequences for the outcomes of portfolio optimization which are not widely recognized by now.

In [6] we consider VCMs that are derived from time-varying beta estimates for mean-variance optimization. When estimating time-varying betas we rely on a time-varying market model given by

$$
y_{i, t}=\alpha_{i, t}+\beta_{i, t} x_{t}+u_{i, t}, \quad u_{i, t} \sim N\left(0, \sigma_{u, i}^{2}\right), \mathrm{i}=1 \ldots \mathrm{N}, \mathrm{t}=1 \ldots \mathrm{T},
$$

with $\mathrm{y}_{i, t}$ denoting the return of stock $\mathrm{i}$ at period $\mathrm{t}$ and $\mathrm{x}_{t}$ the market return, respectively. The error term $u_{i, t}$ captures specific risk of stock i measured by the standard deviation $\sigma_{u, i}$, and the slope coefficient $\beta_{i, t}$ measures the stock's return sensitivity with respect to $\mathrm{x}_{t}$. The coefficient $\alpha_{i, t}$ denotes the stock specific return component at time t.

For estimating time-varying coefficients $\beta_{i, t}$ we employ three well established estimation approaches, namely (i) Moving Window Least Squares (MWLS); (ii) Flexible Least Squares (FLS) and (iii) the Random Walk Model (RWM). See [11] and [15] for an illustration of the estimation methods. We compare estimation results of these approaches with those, generated by the time-invariant Recursive Least Squares-approach (RLS).

Our empirical findings for the U.S. suggest that betas, stock correlations and, hence, VCMs are subject to significant variation in the short run as well as in the long run. In fact, important benefits arise from time-varying estimation of the market model when compared to time-invariant estimation via RLS.

Moreover, we examine the outcomes from mean-variance portfolio selection strategies based on variance-covariance matrices derived from these estimates. We obtain improved ex-ante risk estimates as well as portfolios that have superior risk and return characteristics while being well diversified. For the estimation techniques considered in this paper, we find the same ranking for nearly all investigated criteria. Due to our results, FLS is the best method. It is followed by RWM, MWLS and RLS. The FLS procedure delivers the most precise beta estimates as well as the most precise portfolio risk estimates. Moreover, efficient frontiers suggest higher returns for given volatilities, trading strategies show the highest Sharpe Ratios and finally, portfolios are the most diversified.

To summarize, the portfolio performances found in our empirical analysis indicate a strong need for the application of time-varying estimation approaches for estimating correlations in risk analysis and portfolio construction. Due to our results, the FLS estimate is the favourable method to do so. 


\section{References}

[1] Best, M.J. and Grauer, R.R. (1991) On the Sensivity of Mean-VarianceEfficient Portfolios to Changes in Asset Means: Some Analytical and Computational Results. Journal of Financial Studies 4, 2, 315-342.

[2] Bos, T. and Newbold, P. (1984) An Empirical Investigation of the Possibility of Systematic Stochastic Risk in the Market Model. Journal of Business 57, 35-41.

[3] Chan, L.K.C., Karceski, J. and Lakonishok, J. (1999) On Portfolio Optimization: Forecasting Covariances and Choosing the Risk Model. The Review of Financial Studies 5, 937-974.

[4] Chopra, Kjay K. and William Z. Zimba (1993) The Effect of Errors in Means, Variances and Covariances on Optimal Portfolio Choice. The Journal of Portfolio Management, Winter 1993, 6-11.

[5] Collins, D.W., Ledolter, J. and Rayburn, J. (1987) Some further Evidence on the Stochastic Properties of Systematic Risk. Journal of Business 60, 425-448.

[6] Ebner, Markus and Thorsten Neumann (2008) Time-Varying Factor Models for Equity Portfolio Construction. The European Journal of Finance 14, 381-395.

[7] Ebner, Markus and Thorsten Neumann (2005) Time-Varying Betas of German Stock Returns. Journal of Financial Markets and Portfolio Management. 19, 1, 29-46.

[8] Fabozzi, F.J. and Francis, J.C. (1978) Beta as a Random Coefficient. Journal of Financial and Quantitative Analysis 13, 101-115.

[9] Jacquier, E. and Marcus, A.J. (2001) Asset Allocation Models and Market Volatility. Financial Analysts Journal, 16-29.

[10] Jagannathan, R. and Ma, T. (2003) Risk Reduction in Large Portfolios: Why Imposing the Wrong Constraints Helps. Journal of Finance 58, 1651-1683.

[11] Kalaba, Robert E. and L. Tesfatsion (1989) Time-Varying Linear Regression via Flexible Least Squares. Computers and Mathematics with Applications 17, 1215-1245.

[12] Kim, D. (1993) The Extent of Non-Stationarity of Beta. Review of Quantitative Finance and Accounting 3, 241-254.

[13] Ledoit, Ollivier and Michael Wolf (2002) Improved Estimation of the Covariance Matrix of Stock Returns With an Application to Portfolio Selection. Working paper. University of California, Los Angeles.

[14] Lintner (1965) The Valuation of Risk Assets and the Selection of Risky Investments in Stock Portfolios and Capital Budgets. Review of Economics and Statistics 47, 13-37.

[15] Neumann, T. (2003) Time-Varying Coefficient Models: A Comparison of Alternative Estimation Strategies. Allgemeines Statistisches Archiv 87, 257-281. 
[16] Schwert, G.W. and Seguin, P.J. (1990) Heterscedasticity in Stock Returns. Journal of Finance 45, 1129-1155.

[17] Sharpe, C. (1964) Capital Asset Prices: A Theory of Market Equilibrium under Conditions of Risk. The Journal of Finance 19, 3, 425-442.

[18] Shukla, R., Trzcinka, C. and Winston, K. (1995) Prediction Portfolio Variance: Firm Specific and Macroeconomic Factors. Working Paper, http://ssrn.com/abstract $=6901$.

[19] Sunder, S. (1980) Stationarity of Market Risk: Random Coefficients for Individual Stocks. Journal of Finance 35, 4, 883-896. 